\title{
Block Jacobi relaxation for plane wave discontinuous Galerkin methods
}

\author{
T. Betcke ${ }^{1}$, M.J Gander ${ }^{2}$, and J. Phillips ${ }^{1}$
}

\section{Introduction}

Nonpolynomial finite element methods for Helmholtz problems have seen much attention in recent years in the engineering and mathematics community. The idea is to use instead of standard polynomials Trefftz-type basis functions that already satisfy the Helmholtz equation, such as plane waves [17], Fourier-Bessel functions [8] or fundamental solutions [4]. To approximate the inter-element interface conditions between elements several possibilities exist, such as the ultra-weak variational formulation (UWVF [6]), plane wave discontinuous Galerkin methods (PWDG [15]), partition of unity finite elements (PUFEM [3]), least-squares methods [18, 5], or Lagrange-multiplier approaches [10].

The advantage of Trefftz methods is that they often require fewer degrees of freedom than standard polynomial finite element methods since the basis functions already oscillate with the correct wavenumber. The disadvantage is that the resulting linear systems are often significantly ill-conditioned, making direct solvers or efficient preconditioning for iterative solvers necessary. For very large problems, especially in three dimensions, direct solvers become prohibitively expensive, and preconditioning iterative solvers is a difficult problem for the Helmholtz equation as demonstrated in [9].

Domain decomposition methods, in particular optimized Schwarz methods, have proven to still be effective iterative solvers for finite elements and discontinuous Galerkin methods with polynomial basis functions; for the Helmholtz equation, see $[11,12]$, and for Maxwell's equation, see [1, 7].

In this paper we consider block Jacobi relaxation methods for the PWDG method. In the classical finite element case a block Jacobi relaxation is equivalent to a classical Schwarz method with Dirichlet transmission conditions, see for example [13]. This is however not necessarily the case for discontinuous Galerkin methods, see [14]. We investigate in this short paper what kind of domain decomposition methods one obtains when simply performing a block Jacobi relaxation in a PWDG discretization of the Helmholtz equation, and also show how one can obtain optimized Schwarz methods for such discretizations. Motivated by the block Jacobi relaxation we present a simple algebraic decomposition approach of the system matrix in PWDG methods and demonstrate for an example problem with plane wave basis functions its performance for iterative solvers.

Department of Mathematics, University College London, UK, t.betcke@ucl.ac.uk, joel.m.phillips@gmail.com - Section of Mathematics, University of Geneva, Switzerland, martin.gander@unige.ch 
While in this paper we focus on plane wave basis functions the results are certainly more generally applicable for other Trefftz basis functions, and also for standard polynomial basis functions.

We consider the following model problem: find $u \in \mathscr{C}^{2}(\Omega) \cap H^{1}(\Omega)$, such that

$$
-\Delta u-k^{2} u=f \quad \text { in } \Omega, \quad \frac{\partial u}{\partial n}-S u=g \quad \text { on } \partial \Omega .
$$

Here, $\Omega \subset \mathbb{R}^{d}, d=2,3$, is a bounded domain with Lipschitz boundary $\Gamma:=\partial \Omega$ and $g \in H^{-1 / 2}(\Gamma)$. The operator $S$ is often an exact or approximate Dirichlet to Neumann (DtN) map, e.g. $S=i k$.

We will use the following notation: the triangulation into finite elements of maximum diameter $h$ is denoted by $\mathscr{T}_{h}$. Let $K \in \mathscr{T}_{h}$ be an element of the triangulation. The outward normal direction to $K$ is denoted by $n$. On an edge $e$ between two elements $K^{-}$and $K^{+}$we define for a scalar quantity $v$ the jumps $\llbracket v \rrbracket:=v^{-} n^{-}+v^{+} n^{+}$ and averages $\{\{v\}\}:=\frac{1}{2}\left(v^{-}+v^{+}\right)$. Similarly, for a vector quantity $\sigma$ we define $\llbracket \sigma \rrbracket:=\sigma^{-} \cdot n^{-}+\sigma^{+} \cdot n^{+}$and $\{\{\sigma\}\}:=\frac{1}{2}\left(\sigma^{-}+\sigma^{+}\right)$. On boundary edges we define $[v v]=v \mathbf{n}$ and $\{\{\sigma\}\}=\sigma$.

The set of all interior edges is denoted by $\mathscr{E}^{(i n t)}$ and the set of all eges is denoted by $\mathscr{E}$. Also, let $\tilde{\Omega}$ be defined by $\tilde{\Omega}:=\bigcup_{K \in \mathscr{T}_{h}} K$.

\section{Plane Wave Discontinuous Galerkin Methods}

In the following we give a brief overview of the Plane Wave Discontinuous Galerkin Method (PWDG). For a more detailed introduction and convergence results see $[15,16]$. For each element $K_{i} \in \mathscr{T}_{h}$ we define a local approximation space $V_{i}:=$ $\operatorname{span}\left\{\Psi_{1}^{(i)}, \ldots, \Psi_{N_{i}}^{(i)}\right\}$, where $\Psi_{\ell}^{(i)} \in \mathscr{C}^{2}\left(K_{i}\right) \cap H^{1}\left(K_{i}\right)$ and satisfies $\Delta \Psi_{\ell}^{(i)}+k^{2} \Psi_{\ell}^{(i)}=$ $0, \ell=1, \ldots, N_{i}$. A frequent choice is the plane wave basis set $P W_{i}^{\left(N_{i}\right)}$ defined by $\Psi_{\ell}^{(i)}(x):=e^{i k d_{\ell} \cdot x}$, where the $d_{\ell}$ are direction vectors with $\left\|d_{\ell}\right\|_{2}=1_{2}$. In two dimensions, typically $d_{\ell}=\frac{2 \pi(\ell-1)}{N_{i}}$, that is we take equally spaced directions on the unit circle. In three dimensions several possibilities exist to choose approximately equally spaced directions on the unit sphere (see e.g. [17]). By $V:=\left\{v \in L^{2}(\Omega):\left.v\right|_{K_{i}} \in\right.$ $\left.V_{i} \forall K_{i} \in \mathscr{T}_{h}\right\}$ we denote the global approximation space.

Let $K \subset \mathscr{T}_{h}$. By multiplying (1) with a test function $v \subset V$ on $K$ and integrating by parts we obtain

$$
\int_{K} \nabla u \cdot \overline{\nabla v} d V-k^{2} \int_{K} u \bar{v} d V-\int_{\partial K} \nabla u \cdot n \bar{v} d S=\int_{K} f \bar{v} d V .
$$

A further integration by parts yields

$$
\int_{K} \overline{\left(-\Delta v-k^{2} v\right)} u+\int_{\partial K} u \cdot \overline{\nabla v \cdot n} d S-\int_{\partial K} \nabla u \cdot n \bar{v} d S=\int_{K} f \bar{v} d V .
$$


Define $\sigma:=\frac{1}{i k} \nabla u$ and note that $-\Delta v-k^{2} v=0$. It follows that

$$
\int_{\partial K} u \cdot \overline{\nabla v \cdot n} d S-i k \int_{\partial K} \sigma \cdot n \bar{v} d S=\int_{K} f \bar{v} d V
$$

Using the DG summation formula, see [2],

$$
\sum_{K \in \mathscr{T}_{h}} \int_{\partial K} q_{K} \phi_{K} \cdot n_{K}=\int_{\mathscr{E}} \llbracket[q] \cdot\{\{\phi\}\} d S+\int_{\mathscr{E}(\text { int })}\{\{q\}\}[[\phi] d S
$$

where $q$ is a scalar and $\phi$ a vector quantity we obtain

$$
\begin{gathered}
\int_{\mathscr{E}}[[u]] \cdot\{\{\overline{\nabla v}\}\} d S+\int_{\mathscr{E}(i n t)}\{\{u\}\}[[\overline{\nabla v}]] d S-i k \int_{\mathscr{E}}\{\{\sigma\}\} \cdot[[\bar{v}]] d S \\
-i k \int_{\mathscr{E}(i n t)}[[\sigma]]\{\{\bar{v}\}\} d S=\int_{\tilde{\Omega}} f \bar{v} d V .
\end{gathered}
$$

We now approximate $u$ and $\sigma$ on the edges in terms of their numerical fluxes $\hat{u}_{h}$ and $\hat{\sigma}_{h}$, defined by

$$
\hat{\sigma}_{h}:=\frac{1}{i k}\left\{\left\{\nabla_{h} u_{h}\right\}\right\}-\alpha\left[\left[u_{h}\right]\right]-\frac{\tau}{i k}\left[\left[\nabla_{h} u_{h}\right]\right], \quad \hat{u}_{h}:=\left\{\left\{u_{h}\right\}\right\}+\tau \cdot\left[\left[u_{h}\right]\right]-\frac{\beta}{i k}\left[\left[\nabla_{h} u_{h}\right]\right]
$$

for interior edges, and by

$$
\hat{\sigma}_{h}:=\frac{1}{i k} \nabla_{h} u_{h}-\frac{(1-\delta)}{i k}\left(\nabla_{h} u_{h}-S u_{h} \mathbf{n}-g \mathbf{n}\right), \hat{u}_{h}:=u_{h}-\frac{\delta}{i k}\left(\nabla_{h} u_{h} \cdot \mathbf{n}-S u_{h}-g\right)
$$

for boundary edges. Choices for the parameters $\alpha, \beta, \tau$ and $\delta$ are discussed in [15]. In particular, it is shown there that with the choice $\alpha=\beta=\delta=0.5, \tau=$ 0 the PWDG is equivalent to the UWVF. By replacing $u$ and $\sigma$ in (2) with their corresponding numerical fluxes, noting that

$$
\left\{\left\{\hat{u}_{h}\right\}\right\}=\hat{u}_{h}, \quad\left\{\left\{\hat{\sigma}_{h}\right\}\right\}=\hat{\sigma}_{h}, \quad\left[\left[\hat{u}_{h}\right]\right]=\left[\left[\hat{\sigma}_{h}\right]\right]=0,
$$

on interior edges and using $\left[\left[\hat{u}_{h}\right]\right]=\hat{u}_{h} \mathbf{n},\left\{\left\{\sigma_{h}\right\}\right\}=\hat{\sigma}_{h}$ on boundary edges we arrive at the following variational problem: find $u_{h} \in V$, such that

$$
a\left(u_{h}, v_{h}\right)=\ell\left(v_{h}\right)-b\left(g, v_{h}\right) \quad \forall v_{h} \in V,
$$

where 


$$
\begin{aligned}
& a\left(u_{h}, v_{h}\right):=\int_{\mathscr{E}(e x t)} u_{h} \overline{\nabla_{h} v_{h} \cdot \mathbf{n}} d S-\frac{\delta}{i k} \int_{\mathscr{E}(e x t)} \nabla_{h} u_{h} \cdot \mathbf{n} \overline{\nabla_{h} v_{h} \cdot \mathbf{n}} d S+\frac{\delta}{i k} \int_{\mathscr{E}(e x t)} S u_{h} \overline{\nabla_{h} v_{h} \cdot \mathbf{n}} d S \\
& +\int_{\mathscr{E}_{(i n t)}}\left\{\left\{u_{h}\right\}\right\}\left[\left[\overline{\nabla_{h} v_{h}}\right]\right] d S+\int_{\mathscr{E}_{(i n t)}} \tau \cdot\left[\left[u_{h}\right]\right]\left[\left[\overline{\nabla_{h} v_{h}}\right]\right] d S-\frac{\beta}{i k} \int_{\mathscr{E}_{(i n t)}}\left[\left[\nabla_{h} u_{h}\right]\right]\left[\left[\overline{\nabla_{h} v_{h}}\right]\right] d S \\
& \left.-\delta \int_{\mathscr{E}(\text { ext })} \nabla_{h} u_{h} \cdot \mathbf{n} \overline{v_{h}} d S-(1-\delta) \int_{\mathscr{E}(\text { ext })} S u_{h} \overline{v_{h}} d S-\int_{\mathscr{E}(i n t)}\left\{\left\{\nabla_{h} u_{h}\right\}\right\} \cdot[\bar{v}]\right] d S \\
& +\alpha i k \int_{\mathscr{E}(i n t)}\left[\left[u_{h}\right]\right] \cdot \llbracket \overline{v_{h}} \rrbracket d S+\int_{\mathscr{E}(i n t)} \llbracket\left[\nabla_{h} u_{h}\right] \tau \cdot\left[\left[\overline{v_{h}} \rrbracket d S,\right.\right. \\
& b\left(g, v_{h}\right):=\frac{\delta}{i k} \int_{\mathscr{E}(\text { ext })} g \overline{\nabla v_{h} \cdot \mathbf{n}} d S-(1-\delta) \int_{\mathscr{E}(\text { ext })} g \overline{v_{h}} d S, \\
& \ell\left(v_{h}\right):=\int_{\tilde{\Omega}} f \overline{v_{h}} d V .
\end{aligned}
$$

\section{A natural Schwarz iteration for the UWVF}

In this section we show that a simple block relaxation of the UWVF gives rise to a Schwarz algorithm with Robin transmission conditions, and not the classical Schwarz algorithm with Dirichlet transmission conditions. We consider a simple example problem of a domain $\Omega$ decomposed into two subdomains $\Omega_{1}$ and $\Omega_{2}$ with interface $\Gamma_{12}=\overline{\Omega_{1}} \cap \overline{\Omega_{2}}$. We start by defining the following optimized Schwarz iteration with Robin transmission conditions and optimization parameter $p$ :

$$
\begin{aligned}
-\Delta u_{1}^{(n+1)}-k^{2} u_{1}^{(n+1)} & =f & & \text { in } \Omega_{1}, \\
-\Delta u_{2}^{(n+1)}-k^{2} u_{2}^{(n+1)} & =f & & \text { in } \Omega_{2}, \\
\frac{\partial u_{1}^{(n+1)}}{\partial n_{1}}+p u_{1}^{(n+1)} & =\frac{\partial u_{2}^{(n)}}{n_{1}}+p u_{2}^{(n)} & & \text { on } \Gamma_{12}, \\
\frac{\partial u_{2}^{(n+1)}}{\partial n_{2}}+p u_{2}^{(n+1)} & =\frac{\partial u_{1}^{(n)}}{\partial n_{2}}+p u_{1}^{(n)} & & \text { on } \Gamma_{12}, \\
\frac{\partial u_{1}^{(n+1)}}{\partial n_{1}}+i k u_{1}^{(n+1)} & =g & & \text { on } \Gamma \cap \partial \Omega_{1}, \\
\frac{\partial u_{2}^{(n+1)}}{\partial n_{2}}+i k u_{2}^{(n+1)} & =g & & \text { on } \Gamma \cap \partial \Omega_{2} .
\end{aligned}
$$

Discretizing each of the subproblems with the PWDG and UWVF flux parameters, and setting $p=i k$ gives the sequence of discrete equations

$$
\begin{aligned}
& a_{1}\left(u_{h, 1}^{(n+1)}, v_{h}\right)=\ell_{1}\left(v_{h}\right)-b_{\Gamma \cap \partial \Omega_{1}}\left(g, v_{h}\right)-b_{\Gamma_{12}}\left(\frac{\partial u_{2}^{(n)}}{\partial n_{1}}+i k u_{2}^{(n)}, v_{h}\right), v_{h} \in V_{1}^{(h)}, \\
& a_{2}\left(u_{h, 2}^{(n+1)}, v_{h}\right)=\ell_{1}\left(v_{h}\right)-b_{\Gamma \cap \partial \Omega_{2}}\left(g, v_{h}\right)-b_{\Gamma_{21}}\left(\frac{\partial u_{1}^{(n)}}{\partial n_{2}}+i k u_{1}^{(n)}, v_{h}\right), v_{h} \in V_{2}^{(h)} .
\end{aligned}
$$

Theorem 1. A classical block-Jacobi relaxation applied to the global variational problem (5) discretized with PWDG and UWVF flux parameters, i.e. setting 


$$
\begin{aligned}
& \hat{\sigma}_{1}^{n+1} \cdot \mathbf{n}_{1}=\frac{1}{i k}\{\{\nabla u\}\}^{n+1, n} \cdot \mathbf{n}_{1}-\frac{1}{2}[[u]]^{n+1, n} \cdot \mathbf{n}_{1}, \\
& \hat{\sigma}_{2}^{n+1} \cdot \mathbf{n}_{2}=\frac{1}{i k}\{\{\nabla u\}\}^{n+1, n} \cdot \mathbf{n}_{2}-\frac{1}{2}[[u]]^{n+1, n} \cdot \mathbf{n}_{2}, \\
& \hat{u}_{1}^{n+1}=\{\{u\}\}^{n+1, n}-\frac{1}{2 i k}[[\nabla u]]^{n+1, n}, \\
& \hat{u}_{2}^{n+1}=\{\{u\}\}^{n+1, n}-\frac{1}{2 i k}[[\nabla u]]^{n+1, n},
\end{aligned}
$$

where

$$
\{\{\nabla u\}\}^{n+1, n}:=\frac{1}{2}\left(\left(\nabla u^{-}\right)^{n+1}+\left(\nabla u^{+}\right)^{n}\right), \quad\left[[u]^{n+1, n}:=\frac{1}{2}\left(\left(u^{-}\right)^{n+1} \mathbf{n}^{-}+\left(u^{+}\right)^{n} \mathbf{n}^{+}\right),\right.
$$

leads precisely to the optimized Schwarz method (6) discretized with PWDG and $U W V F$, provided the optimization parameter is set to $p=i k$.

Proof. The classical Robin condition for the Helmholtz equation in this formulation uses the flux term

$$
\hat{\sigma}_{1}^{n+1}=\frac{1}{i k} \nabla u_{1}^{n+1}-\frac{1}{i k}(1-\delta)\left(\nabla u_{1}^{n+1}+i k u_{1}^{n+1} \cdot \mathbf{n}_{1}-\left(\nabla u_{2}^{n}+i k u_{2}^{n} \cdot \mathbf{n}_{1}\right)\right),
$$

and similarly for the second flux term. We have to show that this is precisely the flux (7) given by natural algebraic relaxation. We calculate

$$
\begin{aligned}
\hat{\sigma}_{1}^{n+1} \cdot \mathbf{n}_{1} & =\frac{\delta}{i k} \nabla u_{1}^{n+1} \cdot \mathbf{n}_{1}-(1-\delta) u_{1}^{n+1}+\frac{1-\delta}{i k} \nabla u_{2}^{n} \cdot \mathbf{n}_{1}+(1-\delta) u_{2}^{n} \\
& =\frac{\delta}{i k}[[\nabla u]]^{n+1, n}-(1-\delta)[[u]]^{n+1, n} \cdot \mathbf{n}_{1}+\frac{1}{i k} \nabla u_{2}^{n} \cdot \mathbf{n}_{1}
\end{aligned}
$$

and choosing $\delta=\frac{1}{2}$, and using the relation

$$
\nabla u_{2}^{n} \cdot \mathbf{n}_{1}=\{\{\nabla u\}\}^{n, n} \cdot \mathbf{n}_{1}-\frac{1}{2}[[\nabla u]]^{n, n}
$$

we obtain

$$
\begin{aligned}
\hat{\sigma}_{1}^{n+1} \cdot \mathbf{n}_{1} & =\frac{1}{2 i k}[[\nabla u]]^{n+1, n}-\frac{1}{2}\left[[u]^{n+1, n} \cdot \mathbf{n}_{1}+\frac{1}{i k}\left(\{\{\nabla u\}\}^{n, n} \cdot \mathbf{n}_{1}-\frac{1}{2}[[\nabla u]]^{n, n}\right)\right. \\
& =\frac{1}{i k}\{\{\nabla u\}\}^{n, n} \cdot \mathbf{n}_{1}-\frac{1}{2}\left[[u]^{n+1, n} \cdot \mathbf{n}_{1}+\frac{1}{2 i k}[[\nabla u]]^{n+1, n}-\frac{1}{2 i k}[[\nabla u]]^{n, n}\right. \\
& =\frac{1}{i k}\{\{\nabla u\}\}^{n+1, n} \cdot \mathbf{n}_{1}-\frac{1}{2}\left[[u]^{n+1, n} \cdot \mathbf{n}_{1}\right.
\end{aligned}
$$

and the proof for $\hat{\sigma}_{1}^{n+1}$ is complete. The proof for the other flux terms follows along the same lines. 
The choice $p=i k$ corresponds to a low frequency approximation of the optimal transmission condition, see for example [11]. Optimized Schwarz methods use however a different value for the complex parameter $p$, in order to obtain fast geometric convergence of the method $[11,12]$. The question is how to modify the natural relaxation in order to obtain an optimized Schwarz method. In the following this is described for the $\hat{\sigma}$-flux parameter. The result for the $\hat{u}$-flux follows similarly.

Theorem 2. Performing the modified algebraic relaxation

$$
\begin{aligned}
& \left.\hat{\sigma}_{1}^{n+1} \cdot \mathbf{n}_{1}=\frac{1}{i k}\{\{\nabla u\}\}^{n+1, n} \cdot \mathbf{n}_{1}-\frac{1}{2} \llbracket u u\right]^{n+1, n} \cdot \mathbf{n}_{1}+\frac{1}{2}\left(1-\frac{p}{i k}\right)\left(u_{1, r}^{n+1}-u_{2}^{n}\right), \\
& \hat{\sigma}_{2}^{n+1} \cdot \mathbf{n}_{2}=\frac{1}{i k}\{\{\nabla u\}\}^{n+1, n} \cdot \mathbf{n}_{2}-\frac{1}{2}[u u]^{n+1, n} \cdot \mathbf{n}_{2}+\frac{1}{2}\left(1-\frac{p}{i k}\right)\left(u_{2, l}^{n+1}-u_{1}^{n}\right),
\end{aligned}
$$

where we needed to introduce for subdomain $\Omega_{1}$ the additional variable $u_{1, r}^{n+1}$ to represent the quantity from the other side of the interface corresponding to $u_{2}$, and on $\Omega_{2}$ the additional variable $u_{2, l}^{n+1}$ which represents the quantity from the other side of the interface corresponding to $u_{1}$, we obtain a discretization of the transmission conditions

$$
\begin{aligned}
& \frac{\partial u_{1}^{(n+1)}}{\partial n_{1}}+p u_{1}^{(n+1)}=\frac{\partial u_{2}^{(n)}}{\partial n_{1}}+p u_{2}^{(n)} \\
& \frac{\partial u_{2}^{(n+1)}}{\partial n_{2}}+p u_{2}^{(n+1)}=\frac{\partial u_{1}^{(n)}}{\partial n_{2}}+p u_{1}^{(n)}
\end{aligned}
$$

Proof. With the new variables before relaxation, we can write the flux at the interface as

$$
\hat{\sigma}_{1}^{n+1}=\frac{1}{i k} \nabla u_{1}^{n+1}-\frac{1}{i k}(1-\delta)\left(\nabla u_{1}^{n+1}+i k u_{1}^{n+1} \cdot \mathbf{n}_{1}-\left(\nabla u_{1, r}^{n+1}+i k u_{1, r}^{n+1} \cdot \mathbf{n}_{1}\right)\right) .
$$

In order to substitute the Robin condition from the right, we compute from (13) by adding and subtracting the same term

$$
\frac{\partial u_{1, r}^{(n+1)}}{\partial n_{1}}+i k u_{1, r}^{(n+1)}=\frac{\partial u_{2}^{(n)}}{\partial n_{1}}+p u_{2}^{(n)}+(i k-p) u_{1, r}^{(n+1)},
$$

which we insert into the flux to obtain

$$
\begin{aligned}
\hat{\sigma}_{1}^{n+1} \cdot \mathbf{n}_{1} & =\frac{\delta}{i k} \nabla u_{1}^{n+1} \cdot \mathbf{n}_{1}-(1-\delta) u_{1}^{n+1}+\frac{1-\delta}{i k} \nabla u_{2}^{n} \cdot \mathbf{n}_{1}+(1-\delta) \frac{p}{i k} u_{2}^{n}+(1-\delta) \frac{i k-p}{i k} u_{1, r}^{n+1} \\
& =\frac{\delta}{i k} \llbracket\left[\nabla u \rrbracket^{n+1, n}-(1-\delta) \llbracket u\right]^{n+1, n} \cdot \mathbf{n}_{1}+\frac{1}{i k} \nabla u_{2}^{n} \cdot \mathbf{n}_{1}+\frac{1}{2}\left(1-\frac{p}{i k}\right)\left(u_{1, r}^{n+1}-u_{2}^{n}\right) \\
& =\frac{1}{i k}\{\{\nabla u\}\}^{n+1, n} \cdot \mathbf{n}_{1}-\frac{1}{2} \llbracket[u]^{n+1, n} \cdot \mathbf{n}_{1}+\frac{1}{2}\left(1-\frac{p}{i k}\right)\left(u_{1, r}^{n+1}-u_{2}^{n}\right),
\end{aligned}
$$

where we used the same simplification as in the proof of Theorem 1 to complete the proof for $\hat{\sigma}_{1}^{n+1}$. The proof for $\hat{\sigma}_{2}^{n+1}$ is analogous. 

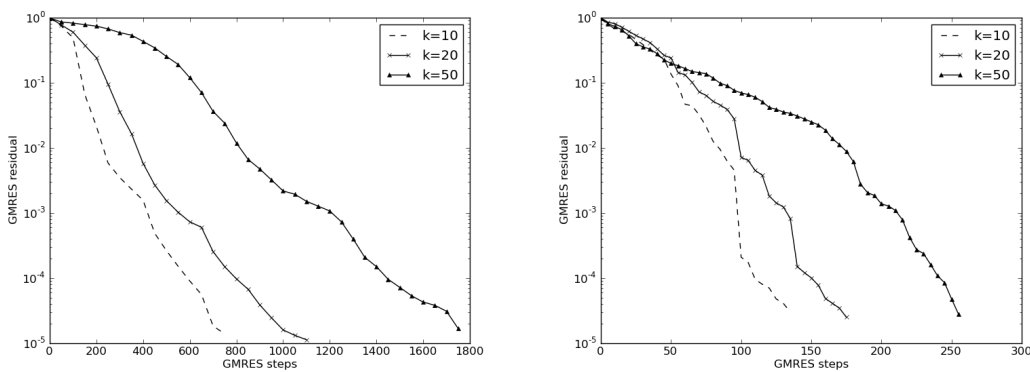

Fig. 1 Left: Convergence of GMRES for the solution of (15) for various wavenumbers $k$. Right: GMRES convergence for the solution of (16) for various $k$.

\section{Discrete system and numerical results}

In this section we present preliminary results for the natural decomposition according to Theorem 1. Results for optimized flux parameters are in preparation. We consider as example a problem partitioned into two subdomains. The global system matrix can be decomposed in the following form.

$$
\left[\begin{array}{cccc}
A_{e_{1}, e_{1}} & A_{e_{1}, e_{2}} & A_{e_{1}, i_{1}} & 0 \\
A_{e_{2}, e_{1}} & A_{e_{2}, e_{2}} & 0 & A_{e_{2}, i_{2}} \\
A_{i_{1}, e_{1}} & 0 & A_{i_{1}, i_{1}} & 0 \\
0 & A_{i_{2}, e_{2}} & 0 & A_{i_{2}, i_{2}}
\end{array}\right]\left[\begin{array}{l}
u_{e_{1}} \\
u_{e_{2}} \\
u_{i_{1}} \\
u_{i_{2}}
\end{array}\right]=\left[\begin{array}{l}
g_{e_{1}} \\
g_{e_{2}} \\
g_{i_{1}} \\
g_{i_{2}}
\end{array}\right] .
$$

Here, $e_{1}$, and $e_{2}$ denote degrees of freedom associated with the interface elements from both sides, and $i_{1}$ and $i_{2}$ denote the interior degrees of freedom. Assume that a fast direct solver is available on each subdomain. Eliminating interior degrees of freedom we arrive at the Schur complement system

$$
\left[\begin{array}{cc}
A_{e_{1}, e_{1}}-A_{e_{1}, i_{1}} A_{i_{1}, i_{1}}^{-1} A_{i_{1}, e_{1}} & A_{e_{1}, e_{2}} \\
A_{e_{2}, e_{1}} & A_{e_{2}, e_{2}}-A_{e_{2}, i_{2}} A_{i_{2}, i_{2}}^{-1} A_{i_{2}, e_{2}}
\end{array}\right]\left[\begin{array}{l}
u_{e_{1}} \\
u_{e_{2}}
\end{array}\right]=\left[\begin{array}{l}
g_{e_{1}} \\
g_{e_{2}}
\end{array}\right]-\left[\begin{array}{c}
A_{e_{1}, i_{1}} A_{i_{1}, i_{1}}^{-1} g_{i_{1}} \\
A_{e_{2}, i_{2}} A_{i_{2}, i_{2}}^{-1} g_{i_{2}}
\end{array}\right] .
$$

From Theorem (1) it follows that a classical block Jacobi method applied to (15) recovers the Schwarz iteration with Robin transmission conditions for the case $p=i k$. Instead of iterating this system via block Jacobi we apply a Krylov subspace iteration and demonstrate the performance of this simple algebraic decomposition at the example of the solution of a Helmholtz equation $-\Delta u-k^{2} u=0$ on the unit square $[0,1]^{2}$. The mesh is a regular triangular mesh with 200 elements. The basis on each mesh consists of 16 equally spaced plane wave directions leading to an overall system size of $n=3200$. On the boundary of the domain impedance conditions are applied, such that the exact solution is a Hankel source $H_{0}(k|x-\hat{y}|)$ with $\hat{y}=(-1,-1)$. The GMRES convergence for the solution of the full system (15) 
for various wavenumbers $k$ is shown in the left plot of Figure 1. The convergence tolerance is set to $10^{-5}$. For the simple algebraic decomposition approach in (16) the results become significantly better. The right plot of Figure 1 shows the results for various wavenumbers for the solution of (16). The subdomain solves were performed with UMFPACK as fast sparse direct solver. The overall system size of (16) is $n=320$. As expected the results deteriorate for higher wavenumbers, which is due to $p=i k$ only being a good parameter for low-frequency problems.

\section{References}

1. A. Alonso-Rodriguez and L. Gerardo-Giorda. New nonoverlapping domain decomposition methods for the harmonic Maxwell system. SIAM J. Sci. Comput., 28(1):102-122, 2006.

2. D.N. Arnold, F. Brezzi, B. Cockburn, and L.D. Marini. Unified analysis of Discontinuous Galerkin methods for elliptic problems. SIAM J. Numer. Anal., 39(5):1749-1779, 2002.

3. I Babuska and J M Melenk. The partition of unity method. Intern. J. Numer. Methods Eng., 40(4):727-758, 1997.

4. A H Barnett and T Betcke. Stability and convergence of the method of fundamental solutions for Helmholtz problems on analytic domains. J. Comp. Phys., 227(7003-7026), 2008.

5. A H Barnett and T Betcke. An exponentially convergent nonpolynomial finite element method for time-harmonic scattering from polygons. SIAM J. Sci. Comput., 32(3):1417-1441, 2010.

6. Olivier Cessenat and Bruno Despres. Application of an Ultra Weak Variational Formulation of Elliptic PDEs to the Two-Dimensional Helmholtz Problem. SIAM J. Numer. Anal., 35(1):255299, 1998.

7. V. Dolean, L. Gerardo-Giorda, and M. J. Gander. Optimized Schwarz methods for Maxwell equations. SIAM J. Scient. Comp., 31(3):2193-2213, 2009.

8. Stanley C Eisenstat. On the rate of convergence of the Bergman-Vekua method for the numerical solution of elliptic boundary value problems. SIAM J. Numer. Anal., 11:654-680, 1974.

9. O. Ernst and M.J. Gander. Why it is difficult to solve Helmholtz problems with classical iterative methods. In O. Lakkis I. Graham, T. Hou and R. Scheichl, editors, Numerical Analysis of Multiscale Problems, pages 325-363. Springer Verlag, 2012.

10. C Farhat, R Tezaur, and J Toivanen. A domain decomposition method for discontinuous Galerkin discretizations of Helmholtz problems with plane waves and Lagrange multipliers. Intern. J. Numer. Methods Eng., 78(13):1513-1531, 2009.

11. M. J. Gander, F. Magoulès, and F. Nataf. Optimized Schwarz methods without overlap for the Helmholtz equation. SIAM J. Sci. Comput., 24(1):38-60, 2002.

12. Martin J. Gander, Laurence Halpern, and Frédéric Magoulès. An optimized Schwarz method with two-sided robin transmission conditions for the Helmholtz equation. Int. J. for Num. Meth. in Fluids, 55(2):163-175, 2007.

13. M.J. Gander. Schwarz methods over the course of time. ETNA, 31:228-255, 2008.

14. M.J. Gander and S. Hajian. Block Jacobi for discontinuous Galerkin discretizations: no ordinary Schwarz methods. In Domain Decomposition Methods in Science and Engineering XXI, Lect. Notes Comput. Sci. Eng. Springer, 2013. submitted.

15. Claude J Gittelson, Ralf Hiptmair, and Ilaria Perugia. Plane wave discontinuous Galerkin methods: analysis of the h-version. M2AN Math. Model. Numer. Anal., 43(2):297-331, 2009.

16. R Hiptmair, A Moiola, and I Perugia. Plane wave discontinuous Galerkin methods for the 2D Helmholtz equation: analysis of the \$p\$-version. SIAM J. Numer. Anal., 49(1):264-284, 2011.

17. A Moiola, R Hiptmair, and I Perugia. Plane wave approximation of homogeneous Helmholtz solutions. Z. Angew. Math. Phys., 62(5):809-837, July 2011.

18. P Monk and Da-Qing Wang. A least-squares method for the Helmholtz equation. Comput. Methods Appl. Mech. Engrg., 175(1-2):121-136, 1999. 